\title{
Lifeworks college students become Well Connected dental ambassadors
}

Community engagement charity, Well Connected, have recently provided oral health advice and education to a group of eight young people at Dartington-based Lifeworks College through the charity's Dental Ambassador programme.

Delivered over a period of six weeks, the project covered different aspects of oral health with emphasis on the key oral health messages. Through a combination of games and activities designed to help consolidate learning, the students built their knowledge of oral health and the importance of looking after their teeth.

Students were familiarised with a mobile dental chair during the programme, similar to that which they would encounter at a dentist surgery, and introduced to the dental light, equipment and protective wear that they, and the dentist, may wear including disposable gloves, aprons and masks.

Using a simulated teaching head, known as a 'Bob in a Box', enabled the students to 'be the dentist' and see teeth from this unique viewpoint. This module also encouraged students to discuss any concerns and worries they may have about visiting the dentist and the Well Connected team were able to support and address these.

Wendy Smith MBE, Strategic Lead for Community Engagement at Well Connected,

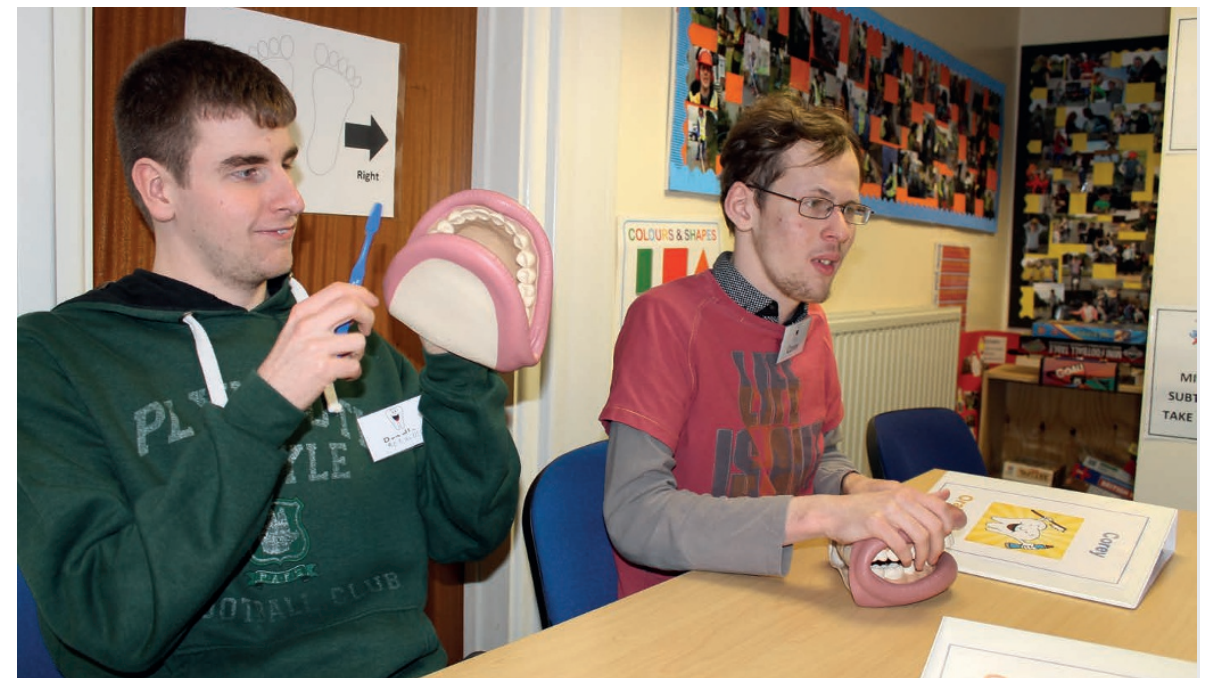

said: 'It's been great to work with the staff and students from Lifeworks College; they are an inspiring group of young people who worked hard during the six week programme. We used a variety of engagement methods to increase knowledge and understanding of oral health, this included creative activities alongside resources, games and dental models.

'A great outcome of the project is that the group took the decision to make some simple swaps to reduce the amount of sugar in some of the food and drinks available to them at college! We are looking forward to working with Lifeworks College again'
The core of Well Connected's Dental Ambassadors programme is empowerment and the students are encouraged to share what they have learnt about oral health and teeth care with their friends and family. At the end of the programme each participant 'graduated' and received a certificate and Dental Ambassador's badge.

Well Connected's mission is to be expert providers in community engagement services for health improvement in the South West region, supporting health improvement agendas and positive lifestyle choices for vulnerable and disadvantaged population groups.

\section{Water fluoridation confirmed to prevent dental decay in US children and adolescents}

A recent study 'Water fluoridation and dental caries in US children and adolescents,' ${ }^{1}$ published in the Journal of Dental Research, evaluated associations between the availability of community water fluoridation and dental caries (decay) experience in US child and adolescent populations.

The study showed that US children and adolescents with greater access to fluoridated drinking water were less likely to experience dental caries. Counties in which over $75 \%$ of the population had access to community water fluoridation saw a $30 \%$ reduction in dental caries experience in the primary dentition, and a $12 \%$ reduction in dental caries experience in the permanent dentition, compared to counties in which less than $75 \%$ had access to community water fluoridation.

The findings are consistent with evidence from the last half-century showing that community water fluoridation continues to provide a substantial dental health benefit for US children and adolescents. The current study boosts the evidence by showing that the benefit is most pronounced early in life, in the primary teeth of 2-8-year-olds.
'This study confirms previously reported findings and provides additional evidence in support of water fluoridation as a core public health intervention promoting oral health,' said Maria Ryan, President of the American Association for Dental Research. 'AADR supports community water fluoridation as a safe and effective, evidence-based intervention for the prevention of dental caries and this report further adds to that evidence base.'

1. Slade G D, Grider W B, Maas W R, Sanders A E. Water Fluoridation and Dental Caries in U.S Children and Adolescents. J Dent Res 2018; DOI: 10.1177/0022034518774331. 